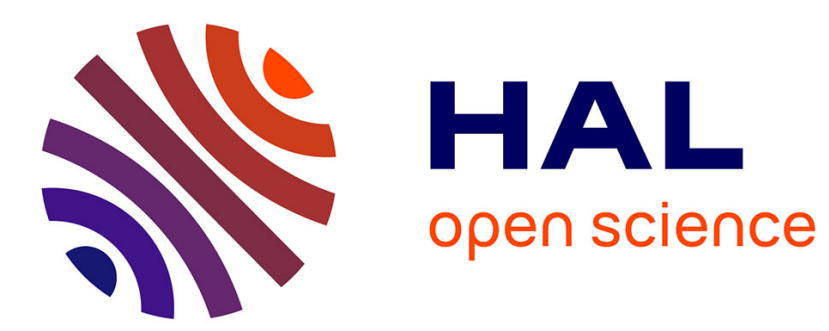

\title{
FLUCTUATIONS AND INTERACTIONS IN THE DILUTE METALLIC STATE
}

\author{
N. Ashcroft
}

\section{To cite this version:}

N. Ashcroft. FLUCTUATIONS AND INTERACTIONS IN THE DILUTE METALLIC STATE. Journal de Physique IV Proceedings, 1991, 01 (C5), pp.C5-169-C5-184. 10.1051/jp4:1991521 . jpa00250645

\section{HAL Id: jpa-00250645 https://hal.science/jpa-00250645}

Submitted on 1 Jan 1991

HAL is a multi-disciplinary open access archive for the deposit and dissemination of scientific research documents, whether they are published or not. The documents may come from teaching and research institutions in France or abroad, or from public or private research centers.
L'archive ouverte pluridisciplinaire HAL, est destinée au dépôt et à la diffusion de documents scientifiques de niveau recherche, publiés ou non, émanant des établissements d'enseignement et de recherche français ou étrangers, des laboratoires publics ou privés. 


\title{
FLUCTUATIONS AND INTERACTIONS IN THE DILUTE METALLIC STATE
}

\author{
N.W. ASHCROFT \\ Laboratory of Atomic and Solid State Physics, Cornell University, and, Laboratoire d'Etudes des Pro- \\ priétés Electroniques des Solides, Grenoble, France
}

\begin{abstract}
The addition of metal to a metal-ammonia solution already in the metallic regime can lead to a further reduction in density. This experimental observation can be accounted for by a straightforward evaluation of the dominant volume-dependent and one-body terms contributing to the thermodynamic functions of the metallic state. Augmenting these terms are smaller two-body terms which are important in a structural sense. Because of the exceptionally low electron-densities found in metal-ammonia solutions, it is proposed that these can be significantly changed, in a state-dependent fashion, by inclusion of fluctuation effects.
\end{abstract}




\section{Introduction}

Among the elements, electron-densities in the metals are often characterized by the linear measure $r_{s}$ defined for a volume $\Omega$ containing $N$ electrons by $\frac{4 \pi}{3} r_{s}^{3} a_{o}^{3}=\left[\frac{1}{\Omega} \int_{\Omega} \overrightarrow{d r}<\right.$ $\left.\hat{\rho}_{e}^{(1)}(r)>\right]^{-1}=\Omega / N$ where $\hat{\rho}_{e}^{(1)}(\vec{r})$ is the one-electron density operator, and $\left\langle\hat{\rho}_{e}^{(1)}(r)>\right.$ its statistical average over the state of the metal in question. Typical values of $r_{s}$ for the metallic elements then lie in the range $0 \leq 2 \leq 6$ the higher values being found for instance in $C s$. The $r_{s}$ range can be extended somewhat by alloying, by compound formation, or even by taking metals to extreme conditions of temperature. But some of the highest values of $r_{s}$ in systems under relatively normal conditions are to be found in the metalammonia solutions. By way of example, the saturated metallic solution of lithium in ammonia $\left(\mathrm{Li}\left(\mathrm{NH}_{3}\right)_{4}\right)$, the lightest of all known non-cryogenic liquids, ${ }^{1}$ has an $r_{s}$ value of $7 \cdot 43$. By systematic reduction of metallic component the system eventually passes into an insulating fluid state, and metal-insulator transition takes place en route; prior to this transition, the $r_{s}$ value has increased still further.

In terms of mean electron densities, these systems are to be considered as extremely dilute. It is interesting that they form at all. From the physical point of view the reason seems to be the following: take a metal atom (say $\mathrm{Li}$ ) in isolation - its valence electron is bound in a pseudopotential, which is attractive and Coulombic at long range (Fig. 1a). But a single electron can also be strongly attracted to an isolated ammonia molecule, the reason for this being immediately attributable to the remarkably large value of its polarizability ${ }^{2}$ $\left(\alpha_{A} \approx 15.27 a_{o}^{3}\right)$ which leads to a law of polarization attraction $\left(\sim-\alpha_{A} e^{2} / r^{4}\right.$, Fig. 1(b)) also at long range. Thus in a mixture of metal atoms and ammonia molecules it can be energetically favorable for the electron to depart the attractive field of the metal ion, to take advantage of polarization attraction of the ammonia molecules, and to be stabilized in part by the ensuing electrostatic (Madelung) energy.

In addition to its role in recording average electron density, the parameter $r_{s}$ also has 
a well understood meaning in terms of the relative importance to be attached to average kinetic energy (proportional to $1 / r_{s}^{2}$ ) and to Coulomb interactions (proportional to $1 / r_{s}$ ). On general grounds it is to be expected that the role of fluctuations in the electron system, either of classical or quantal origin, must rise as average electron density declines. It is for this reason that the metal ammonia solutions may in a real sense be almost ideal systems for the study of the 'simple' low density electron fluid. By 'simple' is meant a system where the electrons originate with s- or p-states; ${ }^{2}$ in particular, it will be argued below that the fluctuational characteristics of the dilute electron fluid, and most especially their manifestation in screening, can lead to power law rather than Thomas-Fermi (or Friedel oscillatory) types of behavior. The forms of such interactions (but not necessarily their magnitudes) are then essentially invariant across the metal-insulator transition.

\section{Characteristics of the Metallic State}

At the temperatures of interest for metal ammonia solutions in the metallic phase, it seems generally agreed that one of the important dynamical units, from the standpoint of subsequent statistical description, is an ion $\left(\mathrm{Li}^{+}\right)$relatively strongly bound to $m(m=4$, apparently) ammonia molecules, each molecule situated with its dipole moment directed outward from the ion. If $c$ is the concentration of metal, then the concentration of molecules not belonging to such units in $c_{A}=(1-(m+1) c) /(1-m c)$ (which is clearly zero at saturation). Thus, in this limited range of the phase diagram the picture is of a metallic fluid composed $N$ complexes in a volume $\Omega$. $\bar{c} N$ are 'free" ammonia molecules; $c N$ are ammonia molecules bound to metallic ions, and hence carrying an overall charge. This is compensated by $c N$ electrons, in volume $\Omega$, in itinerant states. It follows that

$$
(4 \pi / 3) r_{s}^{3} a_{o}^{3}=\frac{1}{c}\left(\frac{\Omega}{N}\right)
$$

Apart from internal binding energies, an approximate ${ }^{3}$ Hamiltonian for this system at the level of pair interactions is

$$
H=\sum_{\alpha}\left\{\hat{T}_{\alpha}+\frac{1}{2} \sum_{\alpha^{\prime}} \int_{\Omega} \overrightarrow{d r} \int_{\Omega} \overrightarrow{d r}^{\prime} v_{\alpha \alpha^{\prime}}\left(\vec{r}-\vec{r}^{\prime}\right) \hat{\rho}_{\alpha \alpha^{\prime}}^{(2)}\left(\vec{r}, \vec{r}^{\prime}\right)\right\}
$$


where $\alpha$ is the species label $(\alpha=e, M, A)$. Thus $\hat{T}_{e}=\sum_{i=1}^{N_{e}}\left(-\hbar^{2} / 2 m_{e}\right) \vec{\nabla}_{i e}^{2}$ is the aggregate of electronic kinetic energies, and so on. In (1) $v_{\alpha \alpha^{\prime}}$ is an interspecies potential (for example $v_{A A}$ is the ammonia-ammonia interaction, $v_{e M}$ is the interaction between an electron and solvated ion, etc.). Finally $\hat{\rho}_{\alpha \alpha}^{(2)}$ is the two-particle density operator

$$
\hat{\rho}_{\alpha \alpha^{\prime}}^{(2)}\left(\vec{r}, \vec{r}^{\prime}\right)=\hat{\rho}_{\alpha}^{(1)}(\vec{r}) \hat{\rho}_{\alpha^{\prime}}^{(1)}\left(r^{\prime}\right)-\delta_{\alpha \alpha^{\prime}} \hat{\rho}_{\alpha}^{(1)}(\vec{r}) \delta\left(\vec{r}-\vec{r}^{\prime}\right)
$$

where the one particle densities are defined by

$$
\hat{\rho}_{\alpha(\vec{r})}^{(1)}=\sum_{i=1}^{N_{\alpha}} \delta\left(\vec{r}-\vec{r}_{i \alpha}\right)
$$

with $N_{\alpha}$ the number of particles of species $\alpha$ (for example, $N_{e}=c N$ ). It should be emphasized that the interactions $v_{\alpha \alpha^{\prime}}$ are not necessarily well known in detail; nevertheless, if they are taken as Fourier transformable, then (1) can be rewritten as

$$
\hat{H}=\sum_{\alpha}\left(\hat{T}_{\alpha}+\frac{1}{2 \Omega} \sum_{\alpha^{\prime}} \sum_{\vec{q}} v_{\alpha \alpha^{\prime}}(-\vec{q}) \hat{\rho}_{\alpha \alpha^{\prime}}^{(2)}(\vec{q},-\vec{q})\right)
$$

or, since long-range interactions are involved $\left(v_{e e}, v_{e M}, v_{M M}\right)$ the $q=0$ terms can be explicitly excerpted to give

$$
\hat{H}=\sum_{\alpha}\left(\hat{T}_{\alpha}+\frac{1}{2 \Omega} \sum_{\alpha^{\prime}} \sum_{q \neq 0} v_{\alpha \alpha^{\prime}}(-\vec{q}) \hat{\rho}_{\alpha \alpha^{\prime}}^{(2)}(\vec{q},-\vec{q})\right)+c N \frac{\alpha_{o}}{r_{s}^{3}}
$$

where $\alpha_{o}$ is proportional to the limit of all $q=0$ residues. ${ }^{4}$ This form is particularly useful in fluid phases since the corresponding statistical averages of $\left\langle\rho_{\alpha \alpha^{\prime}}^{(2)}(\vec{q},-\vec{q})>\right.$ are immediately related to partial structure factors. Given $\hat{H}$ the route to the thermodynamic functions follows as usual from the partition function

$$
Z=\operatorname{Tr} \exp (-\beta \hat{H})
$$

where the trace is to be taken over the combined states of $H$. Invoking adiabatic separation of electron $e$ and other ( $n$, and vastly more massive) degrees of freedom, then (6) can be rewritten

$$
Z=\operatorname{Tr}_{n} e^{-\beta H_{e f f}(\Omega ; \beta)}
$$


where

$$
e^{-\beta H_{e f f}(\Omega, \beta)}=T r_{e(n)} e^{-\beta \hat{H}}
$$

defines an effective Hamiltonian for the metal-ammonia solution in terms of pair potentials and beyond, and which incorporates in a state-dependent fashion the effects of the itinerant electrons. If, as stated previously temperatures are held moderate, then the electrons can be taken close to their ground state, and it then suffices to take a ground state electronic trace

$$
H_{e f f}(\Omega)=<\hat{H}>_{\text {oe }}
$$

with electron coordinates appearing explicitly in 3 of the 6 interaction terms of $\hat{H}$. Equations (5) and (8) are the basis of the subsequent analysis of the thermodynamic functions of the metallic phases of the metal-ammonia systems especially $\mathrm{Li}\left(\mathrm{NH}_{3}\right)_{x}$. They go somewhat beyond the earlier models proposed by Schroeder and Thompson. ${ }^{5}$

\section{Approximate Thermodynamic Functions}

Lacking detailed physical information on 4 of the 6 interactions included in (8), it is not possible at present to give a complete accounting of the thermodynamic functions of metal ammonia solutions. Nevertheless, since electronic and electrostatic energies are dominant, it is possible to identify the origin of certain trends in such systems. Treating the solvated ions $(M)$ and ammonia molecules $(A)$ classically, and also treating the electrons at the level of linear response (it will be argued shortly that at low densities it is necessary to go beyond linear response) we are led to a reasonably acceptable picture based on the proposition that the electronic energy of these systems is a major contributor, overall; the electrostatic energy (from the Madelung sums) is also very significant. Because of this it is plausible to proceed via perturbation theory by associating with $v_{e M}$ and $v_{e A}$ a coupling constant and utilizing the Pauli theorem. The vanishing of the coupling constant corresponds to a physical situation where, with the $q=0$ terms treated as above, an interacting electron gas is present in a uniform background. For paramagnetic arrangements this subsystem 
has an energy per electron (in $R y$ )

$$
\alpha_{K} / r_{s}^{\bar{z}}-\alpha_{e} / r_{s}+\varepsilon_{c}\left(r_{s}\right)
$$

where $\alpha_{K}=2.21$ originates with kinetic energy, $\alpha_{e}=0.916$ with exchange, and the residue $e_{c}\left(r_{s}\right)$ (generally a weak function of density) is the correlation energy. ${ }^{4}$

Through the use of standard response procedures the electron trace required in (6) can be carried out systematically in orders of the coupling constant. By including just linear response in the electron system the procedure leads immediately to one-body energies $\phi_{\alpha}^{(1)}\left(r_{s}\right)$ associated with the agglomeration of electron charge around either the ammonia molecules $(A)$ or the metal-ammonia ions $(M)$; these energies will clearly be proportional to the relative concentrations of each component. In addition, (b), it leads to effective state dependent (screened) two-body pair interactions $\phi_{\alpha \alpha^{\prime}}^{(2)}\left(\vec{r}, r_{s}\right)$ (also, in principle functions of Euler angles). An example of such an effective interaction for spherically averaged $\mathrm{Li}\left(\mathrm{NH}_{3}\right)_{4}^{+}$"ions" is

$$
\phi_{M M}^{(2)}\left(\vec{r}, r_{s}\right)=\frac{1}{(2 \pi)^{3}} \int d \vec{q} e^{-i \vec{q} \cdot \vec{r}}\left(\frac{4 \pi e^{2}}{q^{2}}\right)^{2}\left\{1+\left(\frac{v_{e M}(q)}{4 \pi e^{2} / q^{2}}-\right)^{2}\left(\frac{1}{\epsilon\left(q, r_{s}\right)}-1\right)\right\}
$$

where $\epsilon\left(q, r_{8}\right)$ is the static (state dependent) dielectric function of the interacting electron gas. It is shown in Fig. 2 for the case where $v_{e M}$ is given by an empty-core pseudopotential, with core radius fixed by the minimization procedure discussed below. The important point is that so far as structural rearrangement at fixed density is concerned it leads to energies on the $m R$ scale in some contrast to (8). (As will be seen in a moment, (9) is not necessarily small compared with fluctuation effects arising in the agglomerated electron charge which was considered in the context of the one-body energies.) The fact that the pair terms rise rather steeply at short separation has been used in the past ${ }^{6}$ to establish a variational approach to the evident classical part of the problem namely the determination of the free energy of the $M$ and $A$ subsystems. For example, by introducing a binary hardsphere reference system (with diameters $\sigma_{M}$ and $\sigma_{A}$ ) the Gibbs-Bogolyubov principle can 
be used ${ }^{6}$ to obtain a bound or the Helmholtz energy of the entire system, namely

$$
\begin{aligned}
F(T, \Omega ; c, \bar{c}) & \leq F_{o}\left(T, \Omega_{;} \bar{c} ; \sigma_{A}, \sigma_{M}\right) \\
& +N c\left\{\frac{\alpha_{K}}{r_{s}}-\frac{\alpha_{e M}}{r_{s}}+e_{c}\left(r_{s}\right)\right\} \\
& +\phi_{e M}^{(1)}\left(r_{s}\right)+\phi_{e A}^{(1)}\left(r_{s}\right)+N c \alpha_{o} / r_{s}^{3} \\
& \frac{1}{2 \Omega} \sum_{q \neq 0}\left(S_{M M}\left(q ; \sigma_{M}\right)-1\right) \phi_{M M}^{(2)}(q)+\frac{1}{2 \Omega} \sum_{q \neq 0}\left(S_{A A}\left(q ; \sigma_{A}\right)-1\right) \phi_{A A}^{(2)}(q) \\
& +\frac{1}{2 \Omega} \sum_{q \neq 0}\left(S_{A M}\left(q ; \sigma_{A M}\right)\right) \phi_{A M}^{(2)}(\vec{q}),
\end{aligned}
$$

where $F_{0}$ is the (entropic) free energy of the underlying hard sphere reference system (including rotational contributions) and $S_{\alpha \alpha^{\prime}}^{0}$ are the corresponding structure factors (which may be taken, for example, from the Percus-Yevick approximation ${ }^{7}$ ). In (9) $\alpha_{e M}=\alpha_{e}+\alpha_{M}$ where $\alpha_{M}$ is the Madelung constant for the fluid, a substantial contribution to the energy as noted; and certainly on the scale of $F_{0}$ (per unit).

If accurate expressions for the $\phi_{\alpha \alpha^{\prime}}^{(2)}$ were accessible then at this stage it would be permissible to minimize $F$ for fixed, $T, \Omega, c$, and $\bar{c}$ with respect to the $\sigma_{A}$ and $\sigma_{M}$, resulting in a least upper bound on the free energy. However, such variations are carried out within a structural, and not a cohesive context. For the present (again, lacking detailed knowledge of the $\phi_{\alpha \alpha^{\prime}}^{2}$ ) we will focus on the dominant terms, recognizing however that the entropic contributions are nevertheless crucial in understanding the fluid-fluid phase separation that eventually takes place at lower concentrations. To proceed, consider first the saturated case $\left(\operatorname{Li}\left(N H_{3}\right)_{4}\right)$ where $c=1 / 5 \bar{c}=0$; this will subsequently be diluted $(\bar{c} \neq 0)$ by addition of ammonia. At saturation the internal energy, up to one body embedding energies is

$$
E \approx N c\left\{\frac{\alpha_{K}}{r_{s}^{2}}-\frac{\alpha_{e M}}{r_{s}}+\frac{\bar{\alpha}_{o}\left(r_{s}\right)}{r_{s}^{3}}+e_{c}\left(r_{s}\right)\right\}
$$

where $\bar{\alpha}_{o}\left(r_{s}\right) / r_{s}^{3}$ is the sum of $\phi_{M}^{(1)}\left(r_{s}\right)$ and the $q=0$ residue correction to the Madelung energy discussed above. Since $e_{c}\left(r_{s}\right)$ is a weak function of $r_{s},(11)$ is minimized at a value of $r_{s}$ determined approximately by

$$
\alpha_{e M} r_{s}^{2}-2 \alpha_{K} r_{s}+3 \alpha_{o} \sim 0
$$


But since the minimizing value of $r_{s}$ is known from experiment, $\left(r_{s} \sim 7 \cdot 43\right)$ this establishes $\bar{\alpha}_{o}\left(\bar{\alpha}_{o} \sim 37\right)$. The procedure just described (though it clearly omits structural energies and can be improved by addition of one-body response) is reasonably successful in accounting for the observed equilibrium densities in simple metals and their alloys, and also in widegap semiconductors. Indeed, if in such systems, electron ion pseudopotentials are described by an empty-core form, then the result for $\alpha_{0}$ is quite simple, namely

$$
\alpha_{o}=3 r_{c}^{2}
$$

where $r_{c}$ is the corresponding core size. If a similar stratagem is pursued here (say for $\left.v_{e M}\right)$. Then the "electronic" core size of $\mathrm{Li}\left(\mathrm{NH}_{3}\right)_{4}^{+}$consistent with the observed density is fixed at

$$
r_{c} \approx 3 \cdot 52 a_{o}
$$

The corresponding size of the side of a tetrahedron (with $r_{c}$ as center-to-vertex radius) is about $3 \cdot 1 \AA$. However, since $\bar{\alpha}_{0}$ contains, by definition the effects of dipolar attraction, the corresponding size should be somewhat larger than this. But in any event, the size is reasonably in accord with the value as given by Thompson. ${ }^{1}$

The main point is that the basic length scales of this particular metal follow those of other simple metals, namely the size of the electronic core is considerably smaller than that of the Wigner-Seitz radius; in fact, it is less than half this size. This situation is to be contrasted with the molecular fluids where the equivalent ratio of physical lengths is around $0 \cdot 7 \sim 0 \cdot 8$.

\section{Metallic Dilution}

From the discussion above, it is perhaps in these quintessentially 'metallic' energies that we might seek the major effects of adding unattached (or free) ammonia molecules to the system, obtaining thereby a less than saturated solution. Let $-\alpha_{A}\left(r_{s}\right) / r_{s}^{3}$ be the energy per electron, of embedding an ammonia molecule in an electron gas, whose average density is fixed by $r_{s}$; this is essentially $\phi_{A}^{(1)}$. Using the same approximations as introduced 
above, the energy, per electron is no longer given by (11) but is rather

$$
\begin{aligned}
E / N c=\frac{\alpha_{K}}{r_{s}^{2}}-\frac{\alpha_{e M}}{r_{s}} & +\frac{\bar{\alpha}_{o}\left(r_{s}\right)}{r_{s}^{3}}+e_{c}\left(r_{s}\right) \\
& -\frac{\alpha_{A}\left(r_{s}\right)}{r_{s}^{3}}(1-(M+1) c) / c(1-M c) .
\end{aligned}
$$

It follows that for values of $c$ truly conforming to a metallic state the new minimizing value of $r_{s}$ satisfies

$$
r_{s}=\frac{\alpha_{K}}{\alpha_{e M}}+\sqrt{\left(\frac{\alpha_{K}}{\alpha_{e M}}\right)^{2}+\frac{3 \bar{\alpha}_{o}}{\alpha_{e M}}-\frac{3 \alpha_{c}}{\alpha_{e M}}}
$$

where

$$
\alpha_{c}=\alpha_{A}(1-(m+1) c) / c(1-m c)
$$

From this it also follows that the effects of dilution on the mass density $\rho$, at saturation can be expressed by

$$
\left.\frac{-d \ln \rho}{d \ln c}\right|_{0^{-}}=\frac{r_{A} \alpha_{A}}{r_{s o}\left(\alpha_{e M} r_{s 0}-\alpha_{K}\right)}
$$

where $r_{A}=225 / 2$ and the right hand is then a little less, numerically, than $\alpha_{A}$ itself. Since the latter is, in atomic units, approximately $3 r_{A}^{2}$ where $r_{A}$ is the electronic radius of the ammonia molecule, it follows that the logarithmic derivative in (16) is quite substantial. This may be linked, in part to the extremely steep eutectic seen in this system. Note, however that the addition of metal leads directly by this argument to an expansion of the system, as observed.

This picture must break down for values of $\bar{c}$ that are large simply because the ammonia-ammonia interactions (and ammonia-complex interactions) in the presence of a declining metallic component, themselves become important. However, the exercise can be repeated for the case of a metallic state close to saturation but with additional metal component. Then a similar argument leads to the result

$$
-\left.\frac{d \log \rho}{d \log c}\right|_{0^{+}}=-\frac{\alpha_{i}}{\left(\alpha_{e M} r_{s o}-d_{K}\right) r_{s o}}
$$


where $\alpha_{i}=3 r_{i}^{2}$ is determined by the ionic radius of the metal. From (17) and (16), it follows that

$$
-\frac{(d \log \rho / d \log c)_{0^{-}}}{(d \log \rho / d \log c)_{0^{+}}}=\left(\frac{r_{A}}{r_{i}}\right)^{2}
$$

and again, this may be reflected in part in the asymmetric form of the eutectic. The major point, however, is that either for ammonia rich or metal rich states, the density changes with appropriate concentration are quite significant. The result expressed by (18) though arrived at through consideration of the metal-ammonia system, is not specific to such systems. It reflects the presence of two microscopic length scales intrinsic to an alloying problem; here the length scales are provided by an ion $\left(\mathrm{Li}^{+}\right)$and an ion complex $\left(\mathrm{Li}\left(\mathrm{NH}_{3}\right)_{4}^{+}\right)$. For any reasonably free electron system in which a basic structural complex (e.g. $\mathrm{Pb} \mathrm{K}_{4}$ ) retains its integrity as a concentration variable is altered, a result of the form of (18) will ensue.

\section{Fluctuations}

It has already been remarked that a standard characteristic of the metallic state in the simpler systems is the gross mismatch between the energy scales typical of cohesion, and those typifying structural rearrangement. The former is essentially determined by the energies included in equations (11) or (14); the latter can be obtained from the response argument given above, and indeed is usually advanced in the context of electronically dense systems i.e. systems for which $r_{s}$ is small. The whole point of metal-ammonia systems is that $r_{s}$ is far from this regime, and in fact is even far beyond the point $\left(r_{s} \sim 5 \cdot 5\right)$ where the electron gas suffers a compressional instability (strictly speaking where the second volume derivative of the ground state energy, in the presence of a supplied positive background, changes sign). A largely classical interpretation of such an occurrence in a canonical system would lead to a mild expectation that there might be present among the particles effective attractive interactions impelling such a collapse, much as one would infer from the observed collapses at vapor-liquid transitions in classical gases. In these systems, the origin of such attractions is well understood. It resides with fluctuations in the electronic 
2 charge, which in the two component view of an element is present as the result of response of the electrons to the nuclear field. In exactly the same way, a simple question can be asked about the fuctuational aspects of the electronic charge built up around $M$ and $A$ in the process of forming the one-body $\left(\phi^{(1)}\right)$ energies. The only difference (but it is a rather crucial one) is that the response charge is not bound, though it is well localized, as one knows from Thomas-Fermi approximation. The question here, for large $r_{s}$ systems, is whether higher order response (fluctuations occurring within linear response charge, for example) can lead to structural interactions $\phi_{\alpha \alpha^{\prime}}^{(2)}$ which are actually more significant than the standard terms typified by (9). A simple dimensional argument shows that this is very likely; and calculation verifies it. ${ }^{8}$ Note first that on a time scale short compared with the characteristic time of the interacting electron gas (i.e. $1 / w_{p}$, where $w_{p}$ is the plasma frequency) the distribution of the electronic charge within the time averaged spherically symmetric arrangement around $A$ or $M$ has a full multipole sequence. Selecting, for example, the dipoles, the standard London argument leads to the expectation of $a-1 / r^{6}$, i.e. power-law, attraction between two such ions, each with their localized screening charge. Given this a certain amount of dimensional refinement is now possible since the interaction must be constructed from $\hbar, m_{e}, r_{s}$ and $e^{2}$. The result is

$$
\phi_{f l}^{(2)}(r, \Omega) \propto-\left(\hbar w_{p}\right)\left(r_{s}^{3}\right)^{2} / r^{6} f\left(r_{s}\right)
$$

where $f\left(r_{s}\right)$ is an undetermined factor which must eventually cut off the interaction at very low densities where collective excitations cease to be well defined. This equation may be compared directly with the standard results for atomically localized charge, namely

$$
\phi_{f l}^{(2)}(r) \propto-\Delta\left(\alpha^{2}\right) / r^{6}
$$

where $\alpha$ is the static polarizability, and $\triangle$ a characteristic excitation energy of the atom. As shown by Langreth and $\operatorname{Vosko}^{\circ}$ a more detailed calculation of the appropriate fluctuational diagram (the first of the "ladder" sequence) leads to

$$
\phi_{f l}^{(2)}(n, \Omega) \sim-\left(\hbar \omega_{p}\right)\left(\left(r_{s}^{3}\right)^{2} / r^{6}\right) \log r .
$$


The key feature of (19) or (20) is the rapid growth of this term with declining density, a trend which is fully understandable since the effect we are dealing with owes its origin entirely to fluctuation physics, and this is usually constrained at high densities by increasing kinetic energies.

As applied to metal ammonia solutions this picture is rather straightforward. Consider first the saturated solution. Around each $\mathrm{Li}\left(\mathrm{NH}_{3}\right)_{4}^{+}$complex is found the expected localized complement of static screening charge (the charge whose assembly resulted in the one-body energy $\left.\phi_{M}^{(1)}\right)$. Correspondingly, there is established a statically derived pair interaction whose form is fixed by (9) or its orientational equivalent. But the suggestion is now being made that a further contribution to the pair interaction arises in lower electron density systems, such as we find here, whose origin lies with fluctuations in the response charge around each "ion". It has been emphasized that this picture appears at first to be similar to the atomic case; however, the presumption is that a metallic state does exist so that the response charge is sensitive to an overall macroscopic boundary condition fixed by the system volume $\Omega$. It follows that the fluctuation contribution $\phi_{f l}^{(2)}$ must be a function of state, and from (20) it would appear that changes in $\phi_{f l}^{(2)}$ can develop quite rapidly as compared with (9). Though the present analysis is not expected to be valid in the critical region, the point can nevertheless be made that the rapidity of variation in the pair-interactions will necessarily influence the analysis of the critical regime and possibly lead to renormalization of the critical exponents.

\section{Polarization and Electron Pairing}

The presence in a metal of a constituent with significant internal electronic dynamics (as revealed through a static polarizability $\alpha$ ) carries with it microscopic implications going beyond energetics and global thermodynamic functions. This can be seen by noting that the argument leading to the polarization attraction $-2 \alpha e^{2} /\left|\vec{r}_{1}\right|^{4}$ of an electron at displacement $\vec{r}_{1}$ the from the center of the source of polarization, can also be repeated for a second electron, at $\vec{r}_{2}$ outside the same center. The pair then have a mutual sep- 
aration $\vec{r}=\vec{r}_{1}-\vec{r}_{2}$. The easiest case to consider takes $\left|\vec{r}_{1}\right|=\left|\vec{r}_{2}\right|$, for which the energy is $e^{2}\left(1 / r-4 \alpha /\left|r_{1}\right|^{4}\right)$, and at this level we can already see from the specific arrangement where the electrons are diametrically opposed that for separations less than a critical value $r \lesssim 4^{11 / 6} \alpha^{1 / 3}$ the pair may actually lower their energy by moving closer together. If the pair is not diametrically opposed, the critical separation is smaller, and there is an obvious limit determined by the exclusionary action of the core itself. Between this limit and the critical values we will expect effective attractive interactions. These must then be averaged over all possible values of $\left|\vec{r}_{1}\right|$ and $\left|\vec{r}_{2}\right|$. Exactly this kind of average is encountered in the case where the separation $\left|\vec{r}_{1}-\vec{r}_{2}\right|$ is macroscopic, and an assembly of polarizable objects is interposed. Then the same physics leads to the familiar net (repulsive) interaction $e^{2} / \epsilon r$ where $\epsilon$ is the static dielectric constant. However, on a microscopic scale (e.g. $1 / k_{F}$ ) the net interaction is not necessarily repulsive. The tendency for electron pairing on the solvated electron side of the metal-insulator transition might be viewed in this light; the clear dependence of $\alpha$ suggests that these microscopic pairing tendencies should be even more pronounced as the $C_{60}$ complexes where static polarizabilities $\sim 10^{2} \AA^{3}$ are expected. And if the polarizable objects are in a crystalline array, then there are added enhancements from coherency leading, for the metallic side of the transition, to electron pairing via polarization waves. This observation leads back to the point made earlier namely that in the pursuit of the understanding of dilute itinerant electron systems and especially their fluctuational characteristics, the metal-ammonia systems are almost ideal vehicles.

\section{Acknowledgments}

This work was supported in part by the National Science Foundation under Grant DMR-9017281. 


\section{References and Footnotes}

1. J.C. Thompson, "Electrons in Liquid Ammonia," (Clarendon Press, Oxford) 1976.

2. Note that he polarizability of $N D_{3}$ is noticeably smaller $\left(11 \cdot 48 a_{o}^{3}\right)$ which suggests that fluctuation based attraction between $\mathrm{NH}_{3}-\mathrm{NH}_{3}, \mathrm{NH}_{3}-\mathrm{ND}_{3}$ and $\mathrm{ND}_{3}-\mathrm{ND}_{3}$ (going at long range as the square of the polarizability) should also differ noticeably. In the absence of other intervening phase transitions (e.g. solidification) such differences could be expected to lead to liquid-liquid phase separation (see, for example, S.M. Foiles and N.W. Ashcroft, J. Chem. Phys. $\underline{75}, 3594$ (1981)).

3. The major assumption made in proposing (1) is that three- and higher-center interactions are being neglected. It is not obvious for highly polarizable objects that this can be justified.

4. See, for example, N.W. Ashcroft and D.C. Langreth, Phys. Rev. $\underline{155}, 682$ (1967).

5. R.L. Schroeder and J.C. Thompson, Phys. Rev. 179, 124 (1969).

6. This method is described in detail in N.W. Ashcroft and D. Stroud, Solid State Physics $\underline{33}, 1$ (1978).

7. J.K. Percus and G.J. Yevick, Phys. Rev. 110, 1 (1958); J.L. Lebowitz, Phys. Rev. $\underline{133}, A 895$ (1964); N.W. Ashcroft and D.C. Langreth, Phys. Rev. 156, 685 (1967).

8. C. Rapcewicz and N.W. Ashcroft, Phys. Rev. B $\underline{44}, 4032$ (1991).

9. D.C. Langreth and S.H. Vosko, Phys. Rev. Lett. $\underline{59}, 497$ (1987).

10. See, for example, P. Damay, F. Leclereq, and P. Chieux, J. Phys. Chem. $\underline{88}, 3734$ (1984). 


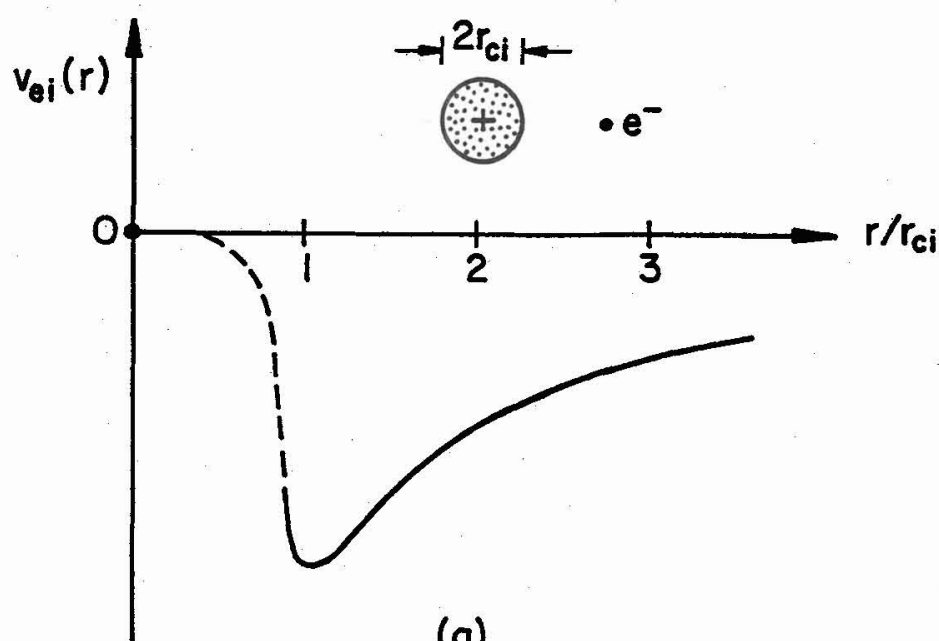

(a)

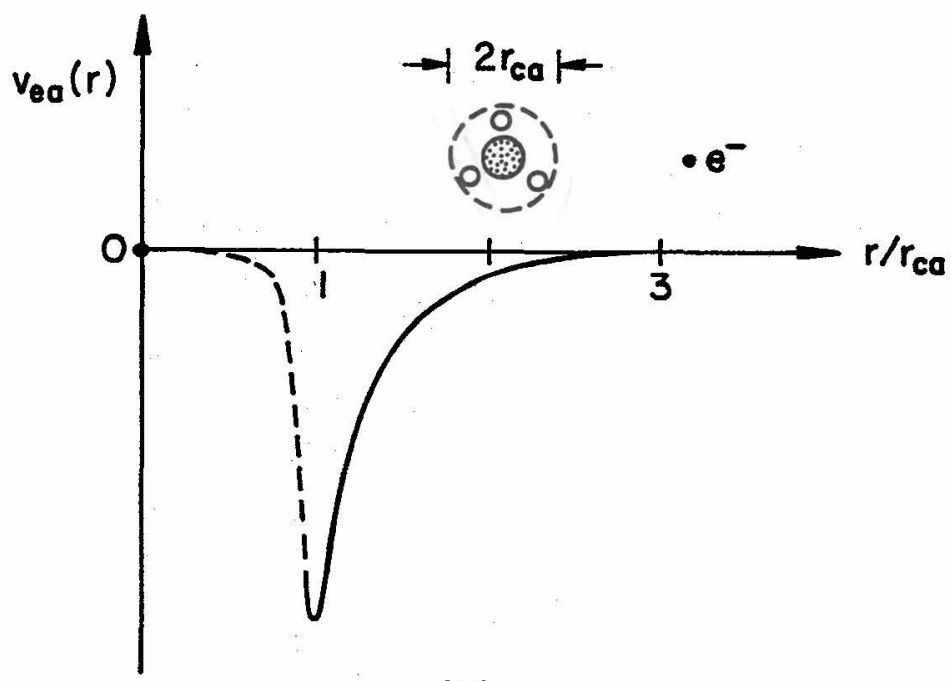

(b)

Figure 1: Ashcroft

Fig. 1: (upper panel) Schematic form of the unscreened electron-ion (ei) pseudopotential for lithium showing the exclusionary core-orthogoralization region for $r / r_{c i}<1$. (lower panel) Schematic form of the core-polarization attraction (ea) of an electron to a neutral ammonia molecule. Again there is an exclusionary region for $r / r_{c a}<1$. Shown here is a spherically averaged version of the true microscopic interaction. 


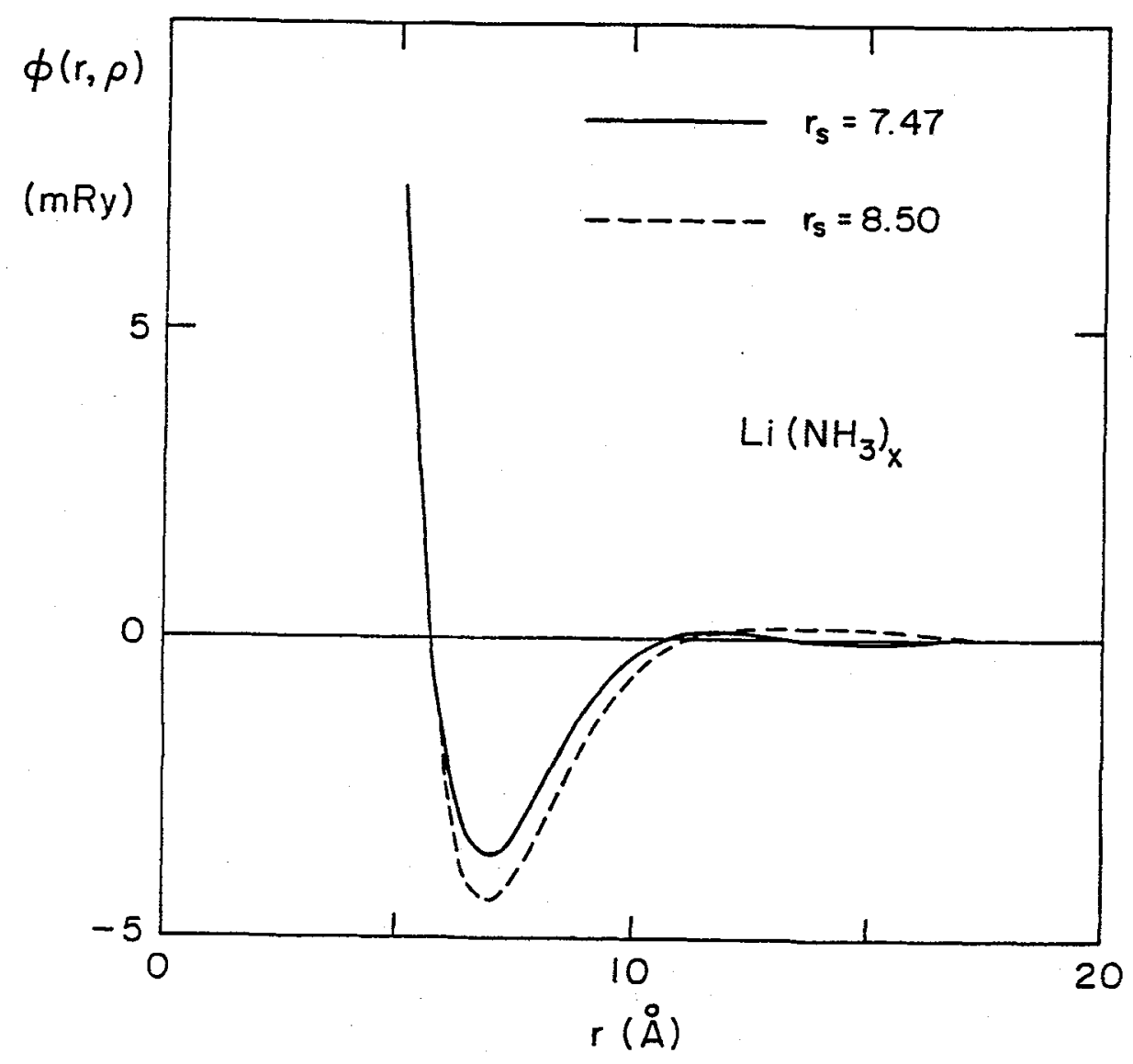

Figure 2: Ashcroft

Fig. 2: Static linear response approximation for the screened, spherically averaged $\mathrm{Li}\left(\mathrm{NH}_{3}\right)_{4}^{+}-$ $\mathrm{Li}\left(\mathrm{NH}_{3}\right)_{4}^{+}$interaction at two densities, indicated by $r_{s}=7 \cdot 47$ and $r_{s}=8 \cdot 50$. Note that the value of $r_{s}=7.47$ corresponds to saturation. 